\title{
Intake of $\alpha$-linolenic acid and other fatty acids in relation to the risk of bladder cancer: results from the New Hampshire case-control study
}

\author{
Maree T. Brinkman ${ }^{1,2 *}$, Margaret R. Karagas ${ }^{3}$, Michael S. Zens ${ }^{3}$, Alan R. Schned ${ }^{4}$, Raoul C. Reulen ${ }^{5}$ \\ and Maurice P. Zeegers ${ }^{2,6}$ \\ ${ }^{1}$ Department of General Practice, Academisch Centrum voor Huisartsgeneeskunde, Katholieke Universiteit Leuven, \\ Kapucijnenvoer 33 Blok J, 3000 Leuven, Belgium \\ ${ }^{2}$ Unit of Urologic and Genetic Epidemiology, Department of Public Health, Epidemiology and Biostatistics, School of \\ Medicine, University of Birmingham, Edgbaston, Birmingham B15 2TT, UK \\ ${ }^{3}$ Section of Biostatistics and Epidemiology, Dartmouth Medical School and Norris-Cotton Cancer Centre, Lebanon, \\ New Hampshire, USA \\ ${ }^{4}$ Department of Pathology, Dartmouth Medical School, Lebanon, New Hampshire, USA \\ ${ }^{5}$ Centre for Childhood Cancer Survivor Studies, School of Health and Population Sciences, University of Birmingham, \\ Birmingham, UK \\ ${ }^{6}$ Department of Complex Genetics, Cluster of Genetics and Cell Biology, NUTRIM School for Nutrition, Toxicology \\ and Metabolism, Maastricht University Medical Center, Maastricht, The Netherlands
}

(Received 24 September 2010 - Revised 6 January 2011 - Accepted 12 February 2011 - First published online 10 May 2011)

\begin{abstract}
The role of dietary fat in bladder cancer aetiology is currently unclear due to few studies, equivocal findings and a lack of information on important dietary fatty acids. The aim of the present study was to investigate the association between the intake of major dietary fats and fatty acids and the risk of bladder cancer. A case-control study was conducted in New Hampshire, USA. Dietary data were collected from 322 cases and 239 controls, and OR and 95\% CI were calculated using unconditional logistic regression. Adjustment was made for potential confounders: sex, age, smoking status, pack-years smoked, cholesterol and energy intake. Statistically significant reduced odds of bladder cancer were observed for high intakes (highest quartile $v$. lowest quartile) of $\alpha$-linolenic acid (ALA) (OR 0.26, 95\% CI 0.10, 0.65; $P$ for trend $=0.01)$ and vegetable fat (OR 0.39, $95 \%$ CI $0 \cdot 18,0 \cdot 86 ; P$ for trend $=0.03)$. Borderline statistically significant reduced odds were detected for polyunsaturated fat (OR 0.43, $95 \%$ CI $0 \cdot 19,0.98 ; P$ for trend=0.07) and linoleic acid (OR 0.43, 95\% CI 0.19, 0.96; $P$ for trend $=0.06$ ). These fats and fatty acids were highly correlated and following adjustment for each other, the only potential inverse association to remain was for ALA. The present findings suggest that ALA may have a protective role against developing bladder cancer; however, further investigation and replication in other epidemiological studies are required. Future research should focus on the type, source and quantities of different dietary fatty acids consumed.
\end{abstract}

Key words: Bladder cancer: $\boldsymbol{\alpha}$-Linolenic acid: Essential fatty acids

Wide variation exists between countries for both the incidence of bladder cancer ${ }^{(1)}$ and the consumption of dietary fat ${ }^{(2)}$. Compared with Asian countries such as Japan, the agestandardised incidence rate of bladder cancer is approximately three times higher in the USA ${ }^{(1)}$. Similarly, the USA, like many Western countries, consumes a diet higher in total fat $(30-40 \% \text { of total dietary intake })^{(3)}$ than the traditional Japanese diet $(<30 \%)^{(4)}$.

While a meta-analysis ${ }^{(5)}$ and systematic review ${ }^{(6)}$ have reported a possible increased risk of bladder cancer associated with a high intake of dietary fat, not all studies have observed such an association ${ }^{(7-9)}$. A possible explanation for inconsistencies in the literature may be due to the fact that dietary fat is obtained from both animal and vegetable or plant sources and comprises more than forty different fatty acids from nature ${ }^{(10)}$. Furthermore, many other fatty acids are produced during food processing and cooking ${ }^{(10)}$. Depending on the chemical structure, there can be different metabolic, physiological and even opposing effects between the various fatty acids ${ }^{(11)}$. It may be that the composition of dietary fat is

Abbreviations: AA, arachidonic acid; ALA, $\alpha$-linolenic acid; EFA, essential fatty acid; LA, linoleic acid; NSAID, non-steroid anti-inflammatory drugs. 
more important in the aetiology of bladder cancer than the actual amount of fat consumed ${ }^{(12)}$. Reports from in vitro ${ }^{(11)}$ and animal studies ${ }^{(13)}$ suggest that the effect of fat on bladder cells can range from anti-carcinogenic to pro-carcinogenic depending on the type of fat/fatty acid involved.

Although not fully understood, one of the mechanisms by which major $n-3$ polyunsaturated fatty acids, EPA and DHA, are reported to possibly inhibit carcinogenesis is through their anti-inflammatory activity ${ }^{(14)}$. While the major n-6 PUFA, linoleic acid (LA), has previously been reported to induce carcinogenesis through oxidative damage and proinflammatory mechanisms, this is not supported by the current evidence from recent human experimental ${ }^{(15)}$ and epidemiological studies ${ }^{(16)}$.

To date, few epidemiological studies ${ }^{(7,12,17,18)}$ have examined the relationship between bladder cancer and the major subgroups of dietary fat. A Serbian case-control study on bladder cancer ${ }^{(19)}$, however, highlighted the importance of considering different dietary sources when it reported a possible inverse association with sunflower oil and positive association with animal fat intake. Similarly, the need to account for the different types of dietary fats in analyses was demonstrated by a Spanish multicentre case-control study $^{(12)}$. The spanish study found that the increased risk of bladder cancer associated with higher intakes of monounsaturated fat disappeared following adjustment for saturated fat. However, quite opposite effects were observed in a Japanese case-control study ${ }^{(18)}$, which has reported inverse associations between bladder cancer risk and both saturated and monounsaturated fat intake.

Even less evidence is available on the association between dietary intake of specific fatty acids and bladder cancer risk. To our knowledge, there is only one such epidemiological study ${ }^{(20)}$, and it has reported no association between bladder cancer overall and intake of $n-3$ marine fatty acids, EPA and DHA.

Due to the current lack of information, the aim of the present study was to investigate major dietary fats and fatty acids to determine whether and to what extent they may influence the risk of bladder cancer. Given the varying physiological functions, it seemed appropriate that some of the key dietary fatty acids be analysed separately.

\section{Methods}

\section{Subjects and methods}

A population-based case-control study was conducted in New Hampshire, USA. Details of the study have previously been reported $^{(21,22)}$. Briefly, bladder cancer cases were identified from the New Hampshire State Department of Health and Human Services Cancer Registry as histologically confirmed, primary bladder cancer diagnosed between 1 July 1998 and 31 December 2001. To be eligible for inclusion in the study, all cases had to be New Hampshire residents aged between 25 and 74 years, had to have a listed phone number and had to speak English. Physician consent was obtained before contacting potential participants. Of the 472 potentially eligible cases who were contacted, a total of 398 (84\%) were interviewed. For efficiency, controls were shared with another study on non-melanoma cancer covering a reference period from 1 July 1997 to 30 March 2000 ${ }^{(23)}$. Controls < 65 years of age were selected from lists obtained from the New Hampshire Department of Transportation. Controls who were 65 years of age and older were chosen from data files provided by the Centers for Medicare and Medicaid Services of New Hampshire. Controls were randomly assigned a comparable reference date corresponding to dates of diagnoses of the case group ${ }^{(24)}$. A total of 526 controls (76\%) were interviewed from a potential 694 confirmed eligible participants ${ }^{(22)}$. The present study was conducted according to the guidelines laid down in the Declaration of Helsinki, and all procedures involving human subjects/patients were approved by the Committee for the Protection of Human Subjects at Dartmouth College. Written informed consent was obtained from all subjects/patients.

\section{Data collection}

Personal interviews were conducted with consenting participants, usually in the homes of the participants. Data were collected on participants' sociodemographic information such as education level, residence, occupation (history), medical history, lifestyle factors (including tobacco smoking), household water supply and family history of cancer. Interviewers were blind to case/control status, and interviews were tape recorded with subjects' consent $(<5 \%$ refused to have the interview taped) to ensure consistent quality of the interview and clarification of details. Cases were also asked whether they held a driver's licence or a Medicare enrolment card for comparability with controls.

\section{Dietary data}

The majority of interviews that included dietary assessment took place between 2000 and 2003 for both cases and controls (for $95 \%$ of subjects). This left data unavailable for 150 cases and 455 controls; a total of 322 cases and 239 controls were included in the dietary analyses of the present study. Subjects from whom dietary data were collected were comparable with those who did not provide dietary data with respect to age, sex and smoking history (data not shown).

Dietary information was collected for the 12-month period before the reference date using a detailed semi-quantitative FFQ originally developed by Willett et al. ${ }^{(25)}$ for the Nurses' Health Study. It contained 121 food items assigned to the following broad categories: dairy; fruit; vegetables; eggs and meat; breads and baked goods. Within these categories, there were specific foods that are rich sources of both animal fats (beef, lamb, pork, poultry, fish, eggs, milk, yogurt, cheese and butter) and vegetable fats (margarine, nuts, salad dressing, soyabeans, vegetable oils, leafy green vegetables and cereals). The FFQ also included a list of beverages, common mineral and vitamin supplements including $n$-3 fatty acids, and seven questions relating to fat consumption, e.g. amount of visible fat removed from meat before 
consumption, frequency of fried foods eaten at home and away from home, the type of margarine used and types of fat used for frying/sautéing and baking. Additionally, there were three open-ended questions where subjects could specify the type of fat or oil usually used in cooking, any additional nutritional supplements and food items not already listed in the FFQ.

Frequency options ranged from never to six or more times a day. Nutrient intake was calculated by multiplying frequency of each food item on the FFQ by the nutrient content for the specific portion size ${ }^{(26)}$. Food composition data were based on data from the United States Department of Agriculture ${ }^{(27)}$. The composition of fatty acids (ranging from shortchain SFA to long-chain PUFA) was available in the nutrient database for all foods included in the FFQ except for the few items without fat content such as alcoholic beverages.

\section{Statistical analysis}

We investigated major dietary fats and fatty acids for potential associations with bladder cancer. These included saturated fat (palmitic acid and stearic acid), monounsaturated fat (palmitoleic acid and oleic acid), polyunsaturated fat, $n-3$ PUFA ( $\alpha$-linolenic acid (ALA), EPA and DHA) and $n-6$ PUFA (LA and arachidonic acid (AA)).

Usual dietary intake for the previous 12 months of all of these fats and fatty acids was estimated in $\mathrm{g} / \mathrm{d}$. The data were analysed in quartiles based on the control group distributions. OR and corresponding 95\% CI were calculated using unconditional logistic regression analysis. As cases and controls were not matched for age and sex (as described earlier, due to using a shared control group), age and sex were included in all models as covariates. Adjustment was also made for a number of possible confounding variables including cigarette smoking status (current/non-current smoker), pack-years of cigarette smoking, cholesterol (except vegetable fat) and total energy intake (quartiles for $\mathrm{kJ} / \mathrm{d}$ ). Likelihoodratio tests for linear trend were conducted by assigning an integer to each quartile of the nutrient, e.g. 1-4, then fitting the term as a continuous variable in the model and comparing the $\log$ likelihood of this model to the log likelihood of a nested model without the nutrient. Potential multicollinearity was assessed by calculating variance inflation factors ${ }^{(28)}$

Interaction was investigated between the intake of ALA and smoking status/history, lipid-soluble antioxidants (vitamin E and total carotenoids) and non-steroid anti-inflammatory drugs (NSAID). In this analysis, smoking status was dichotomised into never/ever smoker, cigarettes/d into high and low categories $(<20 / \geq 20$ cigarettes/d) and duration of smoking $(<27 / \geq 27$ years $)$. ALA and antioxidants were dichotomised into high and low categories based on median values of the controls and NSAID or analgesics based on use before the reference date (no/yes). Participants were classified as having had prior use if they had taken NSAID or other pain medicine at least four times per week for 1 month or longer before the reference date (diagnosis for cases and comparable date randomly assigned to the controls) ${ }^{(22)}$. Drug categories for NSAID/analgesics included phenacetin, paracetamol, ibuprofen and aspirin, and any NSAID (diclofenac, aspirin and ibuprofen $)^{(22)}$. We used the likelihood-ratio test to evaluate interaction, i.e. comparing the log likelihood of regression models with and without the interaction term.

All reported $P$ values were two-sided, and values less than or equal to 0.05 were considered to be statistically significant.

All statistical analyses were performed using the statistical software package Stata/IC 10.1 ${ }^{(29)}$ (StataCorp, College Station, TX, USA).

\section{Results}

Table 1 presents the frequency distributions of selected study characteristics for cases and controls. Approximately three times more men (74\%) than women were bladder cancer cases. A majority of both cases and controls were in the older age category (60 years and over). Compared with controls, there was a higher prevalence of current smokers, a greater number of both cigarettes smoked per day and years smoked among the cases. Cases and controls were comparable for the intake of the major dietary characteristics listed in the table: kJ, total fat, saturated fat, monounsaturated fat, polyunsaturated fat, animal fat and vegetable fat.

The OR and 95\% CI for bladder cancer and intake of major categories of dietary fats and fatty acids are presented in Tables 2 and 3, respectively.

Table 1. Distribution of bladder cancer cases and controls by demographic characteristics, environmental and dietary exposures

(Mean values, standard deviations, number of subjects and percentages)

\begin{tabular}{|c|c|c|c|c|}
\hline & \multicolumn{2}{|c|}{ Cases } & \multicolumn{2}{|c|}{ Controls } \\
\hline & Mean & SD & Mean & SD \\
\hline Total & \multicolumn{2}{|c|}{322} & \multicolumn{2}{|c|}{239} \\
\hline \multicolumn{5}{|l|}{$\operatorname{Sex}^{\star}$} \\
\hline \multicolumn{5}{|l|}{ Men } \\
\hline$n$ & \multicolumn{2}{|c|}{237} & \multicolumn{2}{|c|}{138} \\
\hline$\%$ & \multicolumn{2}{|c|}{74} & \multicolumn{2}{|c|}{58} \\
\hline \multicolumn{5}{|l|}{ Women } \\
\hline$n$ & \multirow{2}{*}{\multicolumn{2}{|c|}{85}} & \multicolumn{2}{|c|}{101} \\
\hline$\%$ & 26 & & \multicolumn{2}{|c|}{42} \\
\hline Age (years) & $62 \cdot 0$ & $9 \cdot 2$ & $60 \cdot 7$ & $10 \cdot 6$ \\
\hline \multicolumn{5}{|l|}{ Smoking status* } \\
\hline \multicolumn{5}{|l|}{ Never smokers } \\
\hline$n$ & \multicolumn{2}{|c|}{$56 \cdot 0$} & \multicolumn{2}{|c|}{$95 \cdot 0$} \\
\hline$\%$ & \multicolumn{2}{|c|}{$17 \cdot 4$} & \multicolumn{2}{|c|}{$39 \cdot 7$} \\
\hline \multicolumn{5}{|l|}{ Ex-smokers } \\
\hline$n$ & \multicolumn{2}{|c|}{$162 \cdot 0$} & \multicolumn{2}{|c|}{$103 \cdot 0$} \\
\hline$\%$ & \multicolumn{2}{|c|}{$50 \cdot 3$} & \multicolumn{2}{|c|}{$43 \cdot 1$} \\
\hline \multicolumn{5}{|l|}{ Current smokers } \\
\hline$n$ & \multicolumn{2}{|c|}{$104 \cdot 0$} & \multicolumn{2}{|c|}{$41 \cdot 0$} \\
\hline$\%$ & \multicolumn{2}{|c|}{$32 \cdot 3$} & \multicolumn{2}{|c|}{$17 \cdot 2$} \\
\hline Smoking (years) ${ }^{*}$ & $32 \cdot 2$ & $14 \cdot 0$ & $27 \cdot 4$ & $15 \cdot 0$ \\
\hline Cigarettes/d* & $26 \cdot 7$ & $14 \cdot 8$ & $21 \cdot 3$ & $15 \cdot 6$ \\
\hline Energy $(\mathrm{kJ} / \mathrm{d})$ & 8203.5 & $3305 \cdot 1$ & $7890 \cdot 8$ & $2953 \cdot 6$ \\
\hline Total dietary fat $(\mathrm{g} / \mathrm{d})$ & $71 \cdot 8$ & 33.0 & 69.8 & 31.8 \\
\hline Saturated fat $(\mathrm{g} / \mathrm{d})$ & $25 \cdot 3$ & $12 \cdot 6$ & $24 \cdot 0$ & 11.4 \\
\hline Monounsaturated fat $(\mathrm{g} / \mathrm{d})$ & $27 \cdot 2$ & $13 \cdot 1$ & $26 \cdot 5$ & $12 \cdot 9$ \\
\hline Polyunsaturated fat $(\mathrm{g} / \mathrm{d})$ & $12 \cdot 9$ & $6 \cdot 2$ & $13 \cdot 1$ & 6.4 \\
\hline Animal fat $(g / d)$ & $39 \cdot 7$ & $20 \cdot 4$ & $37 \cdot 8$ & $19 \cdot 2$ \\
\hline Vegetable fat $(g / d)$ & $32 \cdot 1$ & $17 \cdot 9$ & $32 \cdot 0$ & $17 \cdot 7$ \\
\hline
\end{tabular}

* Mean values were significantly different between cases and controls $(P<0.05)$. 
Table 2. Major categories of fat and bladder cancer*

(Adjusted odds ratios and $95 \%$ confidence intervals)

\begin{tabular}{|c|c|c|c|c|c|}
\hline Daily intake & Q1 & Q2 & Q3 & Q4 & $P$ for trend \\
\hline Total fat $(\mathrm{g} / \mathrm{d})$ & $13 \cdot 37-45 \cdot 66$ & $45 \cdot 67-64.63$ & $64 \cdot 64-88 \cdot 37$ & $\geq 88.38$ & \\
\hline Cases/controls & $66 / 59$ & $75 / 60$ & $98 / 60$ & $80 / 59$ & \\
\hline OR & 1.00 & 1.48 & $1 \cdot 10$ & 0.44 & 0.13 \\
\hline $95 \% \mathrm{Cl}$ & & $0.75,2.91$ & $0.46,2.63$ & $0.15,1.32$ & \\
\hline Animal fat $(g / d)$ & $6 \cdot 09-24 \cdot 16$ & $24 \cdot 17-33.98$ & $33 \cdot 99-47.20$ & $\geq 47.21$ & \\
\hline Cases/controls & $79 / 58$ & $65 / 61$ & $77 / 59$ & $99 / 60$ & \\
\hline OR & 1.00 & 0.60 & 0.62 & 0.52 & 0.22 \\
\hline $95 \% \mathrm{Cl}$ & & $0.33,1.11$ & $0.30,1.30$ & $0.20,1.33$ & \\
\hline Vegetable fat $(\mathrm{g} / \mathrm{d}) \dagger$ & $5.89-19.54$ & $19 \cdot 55-28 \cdot 2$ & $28 \cdot 3-41 \cdot 20$ & $\geq 41 \cdot 21$ & \\
\hline Cases/controls & $79 / 58$ & $70 / 60$ & $93 / 60$ & $75 / 60$ & \\
\hline OR & 1.00 & 0.72 & 0.72 & 0.39 & 0.03 \\
\hline $95 \% \mathrm{Cl}$ & & $0.40,1.30$ & $0.37,1.41$ & $0.18,0.86$ & \\
\hline Total saturated fat $(\mathrm{g} / \mathrm{d})$ & $4.21-15 \cdot 52$ & $15 \cdot 53-22 \cdot 01$ & $22 \cdot 02-30.58$ & $\geq 30.59$ & \\
\hline Cases/controls & $74 / 58$ & $71 / 60$ & $94 / 61$ & $82 / 59$ & \\
\hline OR & 1.00 & 1.08 & 0.80 & 0.41 & 0.07 \\
\hline $95 \% \mathrm{Cl}$ & & $0.58,2.02$ & $0.36,1.75$ & $0.16,1.09$ & \\
\hline Total monounsaturated fat $(\mathrm{g} / \mathrm{d})$ & $4.94-16.97$ & $16 \cdot 98-23 \cdot 56$ & $23 \cdot 57-34 \cdot 28$ & $\geq 34.29$ & \\
\hline Cases/controls & $66 / 60$ & $65 / 59$ & $106 / 61$ & $81 / 59$ & \\
\hline OR & 1.00 & 1.37 & 1.47 & 0.69 & 0.52 \\
\hline $95 \% \mathrm{Cl}$ & & $0.71,2.62$ & $0.64,3.33$ & $0.25,1.88$ & \\
\hline Polyunsaturated fat (g/d) & $2.07-8.87$ & $8 \cdot 88-11 \cdot 78$ & $11 \cdot 79-16 \cdot 28$ & $\geq 16 \cdot 29$ & \\
\hline Cases/controls & $81 / 59$ & $67 / 59$ & $95 / 61$ & $76 / 59$ & \\
\hline OR & 1.00 & 0.74 & 0.79 & 0.43 & 0.07 \\
\hline $95 \% \mathrm{Cl}$ & & $0.40,1.38$ & $0.39,1.60$ & $0.19,0.98$ & \\
\hline Cholesterol $(\mathrm{mg} / \mathrm{d}) \dagger$ & $56 \cdot 36-172 \cdot 46$ & $172 \cdot 47-230 \cdot 48$ & $230.49-331.02$ & $\geq 331.03$ & \\
\hline Cases/controls & $62 / 59$ & $56 / 61$ & $112 / 60$ & $88 / 59$ & \\
\hline OR & 1.00 & 0.99 & 1.61 & 1.03 & 0.55 \\
\hline $95 \% \mathrm{Cl}$ & & $0.55,1.80$ & $0.89,2.91$ & $0.52,2.03$ & \\
\hline
\end{tabular}

Statistically significant reduced odds of bladder cancer were observed for the highest quartile compared with the lowest quartile of intake for ALA (OR 0.26, 95\% CI 0.10, 0.65; $P$ for trend $=0.01)$ and vegetable fat (OR 0.39, 95\% CI 0.18, 0.86; $P$ for trend=0.03). Borderline statistically significant inverse associations were also observed for LA (OR 0.43, 95\% CI $0.19,0.96 ; P$ for trend $=0.06)$ and polyunsaturated fat (OR $0 \cdot 43,95 \%$ CI $0 \cdot 19,0 \cdot 98$; $P$ for trend=0.07). An inverse association and trend of borderline statistical significance was observed for high intake of polyunsaturated fats according to the $n-6: n-3$ ratio (OR 0.59 , 95\% CI $0.34,1.04 ; P$ for trend $=0.06)$. No statistically significant associations or trends were observed for the extent of disease (non-invasive $v$. invasive disease) for these fats/fatty acids or any of the other dietary fats or fatty acids including total intake of $n-3$ or $n-6$ fatty acids (data not shown).

There was a high correlation between intake of vegetable fat, polyunsaturated fat and LA (vegetable fat and polyunsaturated fat, $r$ 0.88; vegetable fat and LA, $r$ 0.88; LA and polyunsaturated fat, $r$ 0.97). Similarly, intake of ALA was highly correlated with vegetable fat $(r$ 0.76) and polyunsaturated fat $(r$ 0.88). Of these dietary fats and fatty acids, only LA and polyunsaturated fat had a high variance inflation factor value $(>10)$, indicating the presence of multicollinearity (data not shown).

We performed additional adjustment for the correlated fats and fatty acids. The only potential inverse association remaining after these adjustments, although it was borderline statistically significant, was between bladder cancer and intake of ALA (highest $v$. lowest quartile for the intake of ALA: adjusted for vegetable fat (OR $0.32,95 \%$ CI $0 \cdot 11,0 \cdot 91 ; P$ for trend $=0.08$ ); adjusted for polyunsaturated fat (OR 0.30, 95\% CI 0.09, 1.01; $P$ for trend=0.07); adjusted for LA (OR $0 \cdot 30,95 \%$ CI 0.09, 1.01; $P$ for trend=0.08)). All other previous associations with bladder cancer disappeared, including the ratio for the intake of $n-6: n-3$ fatty acids (data not shown).

We did not detect any significant interaction between smoking status (ever/never), number of cigarettes smoked per day, duration of smoking (years), intake of vitamin $\mathrm{E}$ or total carotenoids and ALA. Additionally, there was no evidence of a modifying effect for the use of aspirin, ibuprofen, paracetamol or NSAID or analgesics as a group. However, there were borderline statistically significant lower odds of bladder cancer for high ALA intake among phenacetin users compared with non-users ( $P$ for interaction $=0 \cdot 11$; data not shown).

\section{Discussion}

From our investigations into potential associations between major dietary fats and fatty acids and bladder cancer, we identified a consistent inverse association with the intake of ALA. Our initial analyses detected reduced odds of bladder cancer associated with high intakes of vegetable fat, polyunsaturated fat, LA and possibly the ratio for the intake of $n-6: n-3$ fatty 
Table 3. Major dietary fatty acids and bladder cancer*

(Adjusted odds ratios and 95\% confidence intervals)

\begin{tabular}{|c|c|c|c|c|c|}
\hline Major dietary fatty acids $†$ & Q1 & Q2 & Q3 & Q4 & $P$ \\
\hline \multicolumn{6}{|l|}{ SFA } \\
\hline Palmitic acid $(\mathrm{g} / \mathrm{d}), 16: 0$ & $2 \cdot 42-8 \cdot 6$ & $8 \cdot 7-12 \cdot 20$ & $12 \cdot 21-16 \cdot 86$ & $\geq 16.87$ & \\
\hline Cases/controls & $71 / 58$ & $69 / 61$ & 99/59 & $82 / 60$ & \\
\hline OR & 1.00 & 0.96 & 0.89 & 0.40 & 0.09 \\
\hline $95 \% \mathrm{Cl}$ & & $0.49,1.85$ & $0.38,2.09$ & $0.14,1.15$ & \\
\hline Stearic acid $(\mathrm{g} / \mathrm{d}), 18: 0$ & $1.24-3.87$ & $3.88-5 \cdot 3$ & $5 \cdot 4-8 \cdot 10$ & $\geq 8.11$ & \\
\hline Cases/controls & $67 / 59$ & $58 / 59$ & $109 / 58$ & $84 / 62$ & \\
\hline OR & 1.00 & $1 \cdot 16$ & 1.75 & 0.65 & 0.64 \\
\hline $95 \% \mathrm{Cl}$ & & $0.61,2 \cdot 20$ & $0.80,3.81$ & $0.25,1.74$ & \\
\hline \multicolumn{6}{|l|}{ MUFA } \\
\hline Palmitoleic acid $(\mathrm{g} / \mathrm{d}), 16: 1$ & $0.26-0.90$ & $0.91-1.26$ & $1.27-1.82$ & $\geq 1.83$ & \\
\hline Cases/controls & $74 / 58$ & $63 / 63$ & $95 / 57$ & $87 / 60$ & \\
\hline OR & 1.00 & 0.65 & 0.87 & 0.55 & 0.35 \\
\hline $95 \% \mathrm{Cl}$ & & $0.36,1.20$ & $0.42,1.77$ & $0.22,1.37$ & \\
\hline Oleic acid $(\mathrm{g} / \mathrm{d}), 18: 1$ & $4.47-15.33$ & $15 \cdot 34-21.5$ & $21 \cdot 6-30 \cdot 76$ & $\geq 30.77$ & \\
\hline Cases/controls & $66 / 59$ & $69 / 60$ & $101 / 59$ & $82 / 60$ & \\
\hline OR & 1.00 & $1 \cdot 27$ & 1.31 & 0.54 & 0.23 \\
\hline $95 \% \mathrm{Cl}$ & & $0.66,2.44$ & $0.57,2.99$ & $0.20,1.47$ & \\
\hline \multicolumn{6}{|l|}{ PUFA } \\
\hline \multicolumn{6}{|l|}{$n-3$ fatty acids } \\
\hline$\alpha$-Linolenic Acid (ALA), 18:3 & $0.18-0.86$ & $0.87-1.16$ & $1.17-1.63$ & $\geq 1.64$ & \\
\hline Cases/controls & $81 / 59$ & $64 / 60$ & $112 / 60$ & $62 / 59$ & \\
\hline OR & 1.00 & 0.76 & 0.91 & 0.26 & 0.01 \\
\hline $95 \% \mathrm{Cl}$ & & $0.41,1.41$ & $0.44,1.91$ & $0.10,0.65$ & \\
\hline EPA (g/d), $20: 5$ & $0-0.01$ & $0.02-0.04$ & $0.05-0.10$ & $\geq 0 \cdot 11$ & \\
\hline Cases/controls & $94 / 69$ & $55 / 56$ & $107 / 63$ & $66 / 51$ & \\
\hline OR & 1.00 & 0.70 & 1.38 & 0.91 & 0.65 \\
\hline $95 \% \mathrm{Cl}$ & & $0.40,1.23$ & $0.82,2.32$ & $0.52,1.61$ & \\
\hline $\mathrm{DHA}(\mathrm{g} / \mathrm{d}), 22: 6$ & $0-0.06$ & $0.07-0.12$ & $0.13-0.20$ & $\geq 0.21$ & \\
\hline Cases/controls & $77 / 54$ & $73 / 64$ & $89 / 72$ & $83 / 49$ & \\
\hline OR & 1.00 & 0.87 & 0.94 & $1 \cdot 22$ & 0.48 \\
\hline $95 \% \mathrm{Cl}$ & & $0.50,1.51$ & $0.55,1.63$ & $0 \cdot 68,2 \cdot 21$ & \\
\hline \multicolumn{6}{|l|}{$n-6$ fatty acids $(\mathrm{g} / \mathrm{d})$} \\
\hline Linoleic acid $(\mathrm{g} / \mathrm{d}), 18: 2$ & $1.82-7.37$ & $7.38-9.95$ & $9 \cdot 96-13 \cdot 8$ & $\geq 13.9$ & \\
\hline Cases/controls & $82 / 60$ & $71 / 60$ & $90 / 59$ & $76 / 60$ & \\
\hline OR & 1.00 & 0.77 & 0.79 & 0.43 & 0.06 \\
\hline $95 \% \mathrm{Cl}$ & & $0.42,1.41$ & $0.40,1.56$ & $0.19,0.96$ & \\
\hline Arachidonic acid $(\mathrm{g} / \mathrm{d}), 20: 4$ & $0-0.07$ & $0.08-0.12$ & $0.13-0.17$ & $\geq 0.18$ & \\
\hline Cases/controls & $71 / 60$ & $70 / 52$ & $112 / 74$ & $69 / 53$ & \\
\hline OR & 1.00 & 1.02 & $1 \cdot 17$ & 0.72 & 0.65 \\
\hline $95 \% \mathrm{Cl}$ & & $0.55,1.92$ & $0.58,2.34$ & $0.29,1.78$ & \\
\hline$n-6: n-3$ ratio & $3 \cdot 0-7 \cdot 3$ & $7.4-8.5$ & $8 \cdot 6-10 \cdot 1$ & $\geq 10 \cdot 2$ & \\
\hline Cases/controls & $89 / 59$ & $87 / 60$ & $79 / 59$ & $65 / 60$ & \\
\hline OR & 1.00 & 0.96 & $0 \cdot 80$ & 0.59 & 0.06 \\
\hline $95 \% \mathrm{Cl}$ & & $0.56,1.62$ & $0.47,1.38$ & $0.34,1.04$ & \\
\hline
\end{tabular}

Q, quartiles.

* Adjusted for age, sex, smoking status (current $v$. non-current smoker; pack-years smoked (categories: 0-10, 10-20, 20-30, $30-40,40-50$ and $50+)$ ), cholesterol intake and total energy intake $(\mathrm{Q}, \mathrm{kJ} / \mathrm{d})$.

† Nomenclature of fatty acids, e.g. 4:0, length of the carbon chain (short chain, four carbons) and number of double bonds (0); 18:1 (long chain, eighteen carbons) and one double bond ${ }^{(10)}$.

acids. However, these fats and fatty acids were all highly correlated with each other, and initial associations disappeared following mutual adjustment with the exception of ALA. We did not observe any other potential associations, trends or interactions.

Most of the epidemiological evidence available on the association between dietary fatty acids and the risk of cancer has focused on cancer at other body sites, e.g. colorectal $^{(30)}$, breast $^{(31)}$ and prostate ${ }^{(32)}$. To our knowledge, this is one of the first epidemiological studies to investigate the association between dietary fat intake and bladder cancer that examined the effect of different fatty acids. An earlier study ${ }^{(33)}$ compared the plasma levels of essential fatty acids (EFA) of ninety-eight histologically confirmed bladder cancer patients with 477 healthy controls. It was found that levels for most of the EFA, including LA, AA and metabolites of ALA, were significantly lower for bladder cancer patients compared with the controls.

EFA are PUFA that cannot be synthesised within the body and thus must be obtained from the $\operatorname{diet}^{(34)}$. They have a number of key physiological roles, including the regulation of inflammation, blood clotting and vasoconstriction/vasodilation $^{(3)}$. It has also been reported that EFA deficiency may be 'pro-tumorigenic' in rats ${ }^{(34)}$. As EFA make up a large percentage of the phospholipid fatty acid content of the normal rat urothelial membrane, deficiency in these may increase the susceptibility of the urothelium to carcinogenic agents ${ }^{(34)}$. 
We investigated the effect of three of the major $n$ - 3 fatty acids: ALA (18:3); EPA (20:5); DHA (22:6). There were no existing epidemiological studies from which to compare the inverse association that we observed between intake of ALA and bladder cancer risk. However, consistent with a few previous studies, we found no association between bladder cancer risk and intake of EPA and $\mathrm{DHA}^{(20)}$ or $n$-3 fatty acids generally $^{(35)}$. Although EPA and DHA are obtained from fatty cold water fish, they can also be synthesised to a lesser extent from their precursor, $\operatorname{ALA}^{(14)}$.

Despite reports that major $n-6$ fatty acids, LA and AA, may induce bladder carcinogenesis in cell culture ${ }^{(11)}$, we detected a possible inverse relationship for intake of LA before adjustment for vegetable oil and no association with AA. LA is reportedly the major polyunsaturated fat consumed by American adults, comprising 84-89\% of total energy from polyunsaturated fat compared with $9-11 \%$ for ALA $^{(36)}$. We initially observed reduced odds of bladder cancer with the higher ratio for the intake of $n-6: n-3$ fatty acids; however, any potential association disappeared after adjusting for vegetable fat (data not shown). A recent expert report by the $\mathrm{FAO} / \mathrm{WHO}^{(37)}$ concluded that absolute values of these fatty acids are more relevant than the $n-6: n-3$ ratio in terms of optimal physiological function and health.

Contrary to previous studies ${ }^{(5,6,38)}$, we found no evidence that intake of total fat was associated with increased risk of bladder cancer. Similarly, we did not observe inverse associations between bladder cancer risk and intake of saturated and monounsaturated fat reported in a Japanese case-control study $^{(18)}$ consisting of 297 bladder cancer cases and 295 controls. These major subgroups of fats are available from both vegetable and animal sources, and may have been derived predominantly from vegetable sources in the Japanese study population ${ }^{(10)}$. Interestingly, we did observe a low-point estimate that was of borderline statistical significance for saturated fat, which was highly correlated with palmitic acid $(r$ 0.96), a saturated fat also obtained from plant sources, e.g. palm oil ${ }^{(10)}$. Similarly, our lack of association between the intake of animal fat and bladder cancer risk disagreed with reports of a positive association from a Serbian case-control study ${ }^{(19)}$ of 130 cases and controls. Population differences in the type, composition and quantity of animal fat consumed and even cooking methods may account for the lack of agreement between the Serbian and our American study.

Accurate estimation of dietary intake of specific fats/fatty acids is difficult, particularly for PUFA, like ALA, which are typically consumed in small quantities ${ }^{(16,39)}$. In the absence of biological markers, it is important that dietary assessment instruments and nutrient composition databases can provide adequate estimates of usual dietary intake of these fatty $\operatorname{acids}^{(39)}$. Some earlier studies ${ }^{(40,41)}$ have assessed the performance of dietary measures in estimating fatty acid exposure using biological samples. A cross-sectional study ${ }^{(40)}$ of 276 men and 257 women has reported weak, but significant, correlations between plasma measures of several EFA (LA, AA, EPA and DHA), although not ALA, and dietary intake. $\mathrm{Arab}^{(41)}$ also compared dietary measures of specific fatty acids with the fatty acid content of adipose tissue (as a long-term measure of exposure), and found that there were moderate-to-strong correlations between the two measures for PUFA, including $n-3$ long-chain fatty acids. While we used a validated FFQ that contained a wide range of common food sources for the fats/fatty acids under investigation and a comprehensive nutrient composition database (containing the fatty acid content of several thousand different food items), we do, however, recognise that the present finding may be biased towards the null due to measurement error.

Estimating dietary intake of specific fatty acids is further complicated by the fact that many fatty acids share common foods ${ }^{(10)}$. For example, while olive oil predominantly comprises the MUFA, oleic acid (approximately 70\%), ALA and LA are also present in small quantities ${ }^{(27)}$. We attempted to disentangle the effect of individual fatty acids by adjusting for highly correlated fats/fatty acids. Although this reduced the number of potential inverse associations to just ALA, there is the possibility that this may have led to over adjustment and failure to detect true effects as a consequence. While it is also possible that other dietary components common to a plant-based diet, e.g. carotenoids and vitamin E, may be responsible for the inverse associations that we observed for ALA, we detected no modifying effect by these micronutrients from our analyses.

Another limitation of dietary assessment is the potential for recall bias with a retrospective study design. However, as dietary factors are not widely associated with the risk of bladder cancer ${ }^{(42)}$ and recall of dietary intake is likely to be non-differential between cases and controls, recall bias should minimally affect the present results if at all. Although we cannot totally discount the possibility that there may have been dietary changes due to diagnosis of bladder cancer, it has previously been suggested that pre-clinical disease is unlikely to have a major effect on dietary intake ${ }^{(43)}$. Consistent with this, we observed similar consumption levels of major dietary components, energy and total dietary fat intake for cases and controls and for cases both with and without invasive disease. Additionally, there was no association between intake of potentially protective fats/fatty acids and extent of disease (non-invasive $v$. invasive bladder cancer).

While controls drawn from two sources, the Department of Transportation (for those under 65 years) and Medicare (for those 65 years and older), could be an issue, it is minimised by the fact that these are considered nearly complete sources of records in our population. Among cases, over 95\% report having a driver's licence (for those under 65 years) as do over $95 \%$ (for those 65 years and older) report being enrolled in Medicare (data not shown). Furthermore, we examined the characteristics of controls younger than 65 years and those over 65 years, and found them to be comparable with respect to sex, smoking and medical access variables (i.e. visit to a doctor in the past 2 years); however, older subjects had less than a college education ( $40 \%$ of those under 65 years and $50 \%$ of those 65 years and older). Level of education, however, is not a strong risk factor for bladder cancer (including in our own data) and thus was not considered a potential confounder in our analysis. 


\section{Conclusions}

Dietary fat is a heterogeneous macronutrient, and in order to understand its role in bladder cancer aetiology, the effects of individual fatty acids need to be examined separately. While our observations suggest that ALA may have a protective role against developing bladder cancer, further replication in other studies is required. The present study additionally highlighted the difficulty in measuring dietary fat intake due to the wide range of fatty acids available from both different and shared food sources ${ }^{(10)}$. Overall, the present results indicate that the possible effect of dietary fat on the risk of developing bladder cancer may depend on the type and source of fat as well as on the quantity consumed.

\section{Acknowledgements}

None of the authors has any conflict of interest to declare. The present study was funded in part by grant no. 5 P42 ES007373 from the National Institute of Environmental Health Sciences (NIEHS), National Institutes of Health and CA57494 from the National Cancer Institute, National Institutes of Health. Its contents are solely the responsibility of the authors and do not necessarily represent the official views of the NIEHS, National Institutes of Health. M. P. Z. was partly funded by Cancer Research, UK. M. T. B., M. R. K. and M. P. Z. participated in the conception and design of the study. A. R. S. carried out histopathological reviews. M. S. Z. was involved with the data management. M. T. B. carried out the statistical analysis and drafted the manuscript. M. T. B., R. C. R., M. R. K., M. S. Z., A. R. S. and M. P. Z. participated in the interpretation of results and critical review of several drafts of the manuscript. All authors read and approved the final manuscript. The authors would like to thank the physicians, pathology laboratories, staff members and many participants of the New Hampshire Health Study for making the present study possible.

\section{References}

1. Parkin DM, Bray F, Ferlay J, et al. (2005) Global cancer statistics, 2002. CA Cancer J Clin 55, 74-108.

2. Wolmarans P (2009) Background paper on global trends in food production, intake and composition. Ann Nutr Metab 55, 244-272.

3. World Cancer Research Fund/American Institute for Cancer Research (2007) Fats and oils. In Food, Nutrition, Physical Activity and the Prevention of Cancer: A Global Perspective, pp. 135-140. Washington DC: AICR.

4. Yoneyama S, Miura K, Sasaki S, et al. (2007) Dietary intake of fatty acids and serum C-reactive protein in Japanese. $J$ Epidemiol 17, 86-92.

5. Steinmaus CM, Nunez S \& Smith AH (2000) Diet and bladder cancer: a meta-analysis of six dietary variables. Am J Epidemiol 151, 693-702.

6. Brinkman M \& Zeegers MP (2008) Nutrition, total fluid and bladder cancer. Scand J Urol Nephrol Suppl 218, 25-36.

7. Michaud DS, Spiegelman D, Clinton SK, et al. (2000) Prospective study of dietary supplements, macronutrients, micronutrients, and risk of bladder cancer in US men. $A m$ J Epidemiol 152, 1145-1153.
8. Chyou PH, Nomura AM \& Stemmermann GN (1993) A prospective study of diet, smoking, and lower urinary tract cancer. Ann Epidemiol 3, 211-216.

9. La Vecchia C, Negri E, Decarli A, et al. (1989) Dietary factors in the risk of bladder cancer. Nutr Cancer 12, 93-101.

10. Jones G (1997) Fats. In Food and Nutrition Australasia, Asia and the Pacific, 3rd ed., pp. 205-214 [ML Wahlqvist, editor]. East Melbourne, VIC: Allen \& Unwin.

11. Maggiora M, Bologna M, Ceru MP, et al. (2004) An overview of the effect of linoleic and conjugated-linoleic acids on the growth of several human tumor cell lines. Int J Cancer 112, 909-919.

12. Riboli E, Gonzalez CA, Lopez-Abente G, et al. (1991) Diet and bladder cancer in Spain: a multi-centre case-control study. Int J Cancer 49, 214-219.

13. Cremonezzi DC, Diaz MP, Valentich MA, et al. (2004) Neoplastic and preneoplastic lesions induced by melamine in rat urothelium are modulated by dietary polyunsaturated fatty acids. Food Chem Toxicol 42, 1999-2007.

14. Larsson SC, Kumlin M, Ingelman-Sundberg M, et al. (2004) Dietary long-chain $n-3$ fatty acids for the prevention of cancer: a review of potential mechanisms. Am J Clin Nutr 79, 935-945.

15. Adam O, Tesche A \& Wolfram G (2008) Impact of linoleic acid intake on arachidonic acid formation and eicosanoid biosynthesis in humans. Prostaglandins Leukot Essent Fatty Acids 79, 177-181.

16. Gerber M (2009) Background review paper on total fat, fatty acid intake and cancers. Ann Nutr Metab 55 , $140-161$.

17. Bruemmer B, White E, Vaughan TL, et al. (1996) Nutrient intake in relation to bladder cancer among middle-aged men and women. Am J Epidemiol 144, 485-495.

18. Wakai K, Takashi M, Okamura K, et al. (2000) Foods and nutrients in relation to bladder cancer risk: a case-control study in Aichi Prefecture, Central Japan. Nutr Cancer 38, 13-22.

19. Radosavljevic V, Jankovic S, Marinkovic J, et al. (2005) Diet and bladder cancer: a case-control study. Int Urol Nephrol 37, 283-289.

20. Holick CN, Giovannucci EL, Stampfer MJ, et al. (2006) A prospective study of fish, marine fatty acids, and bladder cancer risk among men and women (United States). Cancer Causes Control 17, 1163-1173.

21. Karagas MR, Tosteson TD, Blum J, et al. (1998) Design of an epidemiologic study of drinking water arsenic exposure and skin and bladder cancer risk in a U.S. population. Environ Health Perspect 106, 1047-1050.

22. Fortuny J, Kogevinas M, Zens MS, et al. (2007) Analgesic and anti-inflammatory drug use and risk of bladder cancer: a population based case control study. BMC Urol, 10 August, 7-13.

23. Applebaum KM, Karagas MR, Hunter DJ, et al. (2007) Polymorphisms in nucleotide excision repair genes, arsenic exposure, and non-melanoma skin cancer in New Hampshire. Environ Health Perspect 115, 1231-1236.

24. Karagas MR, Park S, Warren A, et al. (2005) Gender, smoking, glutathione- $S$-transferase variants and bladder cancer incidence: a population-based study. Cancer Lett 219, 63-69.

25. Willett WC, Sampson L, Stampfer MJ, et al. (1985) Reproducibility and validity of a semiquantitative food frequency questionnaire. Am J Epidemiol 122, 51-65.

26. Michaud DS, Spiegelman D, Clinton SK, et al. (1999) Fruit and vegetable intake and incidence of bladder cancer in a male prospective cohort. J Natl Cancer Inst 91, 605-613. 
27. US Department of Agriculture, Agricultural Research Service (2004) USDA Nutrient Database for Standard Release 16. Washington, DC: US Department of Agriculture.

28. Slinker BK \& Glantz SA (1985) Multiple regression for physiological data analysis: the problem of multicollinearity. Am J Physiol 249, R1-R12.

29. StataCorp (2009) Stata/IC 10.1. College Station TX: StataCorp.

30. Theodoratou E, McNeill G, Cetnarskyj R, et al. (2007) Dietary fatty acids and colorectal cancer: a case-control study. Am J Epidemiol 166, 181-195.

31. Thiébaut AC, Chajès V, Gerber M, et al. (2009) Dietary intakes of omega- 6 and omega-3 polyunsaturated fatty acids and the risk of breast cancer. Int J Cancer 124, 924-931.

32. Simon JA, Chen Y-H \& Bent S (2009) The relation of alphalinolenic acid to the risk of prostate cancer: a systematic review and meta-analysis. Am J Clin Nutr 89, 1558S-1564S.

33. McClinton S, Moffat LE, Horrobin DF, et al. (1991) Abnormalities of essential fatty acid distribution in the plasma phospholipids of patients with bladder cancer. $\mathrm{BrJ}$ Cancer 63, 314-316.

34. Eynard AR (1998) Is the risk of urinary tract tumorigenesis enhanced by a marginal chronic essential fatty acid deficiency (EFAD)? Nutrition 14, 211-216.

35. MacLean CH, Newberry SJ, Mojica WA, et al. (2006) Effects of omega-3 fatty acids on cancer risk: a systematic review. JAMA 295, 403-415.
36. Kris-Etherton P, Taylor DS, Yu-Poth S, et al. (2000) Polyunsaturated fatty acids in the food chain in the United States. Am J Clin Nutr 71, 179S-188S.

37. Smit LA, Mozaffarian D \& Willett W (2009) Review of fat and fatty acid requirements and criteria for developing dietary guidelines. Ann Nutr Metab 55, 44-55.

38. Vena JE, Graham S, Freudenheim J, et al. (1992) Diet in the epidemiology of bladder cancer in western New York. Nutr Cancer 18, 255-264.

39. Carayol M, Grosclaude P \& Delpierre C (2010) Prospective studies of dietary alpha-linolenic acid intake and prostate cancer risk: a meta-analysis. Cancer Causes Control 21, 347-355.

40. Astorg P, Bertrais S, Laporte F, et al. (2008) Plasma $n-6$ and $n-3$ polyunsaturated fatty acids as biomarkers of their dietary intakes: a cross-sectional study within a cohort of middle-aged French men and women. Eur J Clin Nutr 62, $1155-1161$.

41. Arab L (2003) Biomarkers of fat and fatty acid intake. J Nutr 133, 925S-932S.

42. World Cancer Research Fund/American Institute for Cancer Research (2007) Cancers. In Food, Nutrition, Physical Activity, and the Prevention of Cancer: a Global Perspective, pp. 312-314. Washington DC: AICR.

43. Garcia-Closas R, Garcia-Closas M, Kogevinas M, et al. (2007) Food, nutrient and heterocyclic amine intake and the risk of bladder cancer. Eur J Cancer 43, 1731-1740. 